\title{
New ways with crystal growth
}

\section{Computer simulations of the aggregation process are fashionable because the problems are complicated. But mathematics still has a place.}

THAT crystal growth is a complicatea process requires no formal proof: how, otherwise, runs the conventional argument, would snowflakes have such a shape? One consequence of considerations such as these is the wealth of numerical simulations which there have been, in the past few years, of how elementary particulates may be added to the surface of a growing aggregate by diffusion from the exterior. This fashion is a reaction to earlier disappointments, and particularly to the recognition that earlier macroscopic models of the growth of aggregates, which distinguished between the various positions on an extending surface only by their macroscopic properties and especially their curvature, have given only poor accounts of what really happens. So it may be a salutory lesson for all concerned that there should now have appeared an analytical calculation of the changing shape of a surface growing by aggregation; people who have been learning how to program increasingly complicated computer machinery may yet find themselves mugging up on differential equations again.

To be fair, the question that has arisen is only part of the whole complicated problem, and may be put like this. In the earliest stages of the growth of a crystal or other aggregate, chance adhesion will be the rule, so that small particles will tend to be rough particles. Given time to reach equilibrium, of course, the roughnesses will become smooth as particles in exceptional positions find less energetically exposed sites at which to settle down, but there will also be circumstances in which a surface growing at a modest rate will become smoother in the course of time as added particles find their way preferentially to the gaps left in the growing surface. To account for the irregularity of snowflakes, either microscopically (by numerical simulation) or macroscopically (by allowing for the extra energy of elements added at places where the curvature is great using the language of surface tension), boils down to supposing that the circumstances are dynamic, not static. The rapidly growing tip of a snowflake crystal is, because of its geometry, exposed to a greater volume of the environment from which further elements may be accreted than would be a re-entrant part of the snowflake surface, while the speed with which newly accreted elements may readjust their positions is so much less than that with which new elements are added that aberrations are, so to speak, frozen.

The new development is surprisingly straightforward, and the product of the cosmopolitan collaboration of Mehran Kardar of Harvard University, Giorgio Parisi from the University of Rome and Yi-Cheng Zhang from the Brookhaven National Laboratory (Phys. Rev. Lett. 56, $889 ; 1986)$. The question they set out to answer is that of how a surface accreting extra elements will grow in the course of time, allowing that the circumstances are midway between those which determine the behaviour of a growing snowflake, where there is no opportunity for newly added particles to readjust their positions, and those that dominate the macroscopic growth of solids which are predisposed towards the emergence of globules of one form or another.

The simplest starting point is the assumption that the normal to a surface will determine the direction in which it will grow locally. There follows a geometrical construction of what happens at the surface, beginning with the assumption that it is possible to measure the distance of the surface as a function of time from some fixed plane; the distance might, for example, be the height of the surface of a growing crystal from the bottom of a vessel in which it is allowed to settle. At any point, the rate of change of the height with time will therefore be determined by the rate of growth normal to the surface and by the square of the gradient of the surface at that point. From this it follows naturally and very simply that the rate of change with time of height from the reference plane is proportional, first, to the curvature of the surface at that point and, second, to the square of the gradient of the surface at that same point. The first term is that which represents the effect of surface tension. The second, which makes the differential equation non-linear, allows for lateral growth. For completeness, the specification of the problem requires that there should be an element of noisiness, represented by a third terma, in the gaussian function in time and space, whose only important property is that its average should be zero.

Mathematicians will quickly observe that this non-linear equation describing the evolution of an accreting surface can be converted into a linear form that of a diffusion process. Indeed, in this form the equations are similar to those which des- cribe the behaviour of branched polymer molecules in which the surrounding environment discriminates against overextended forms.

The interest of this formulation of the problem of the growth of accreting surfaces is that, in principle, it allows of a global view of what may be happening. If the noisiness of the problem is left out of account, the equations suggest that a onedimensional growing surface will consist of a sequence of parabolic segments which, as time goes on, will grow at each others' expense, the larger segments swallowing the smaller. If the surface is twodimensional, there will be patches on the surface which form a comprehensive network covering the whole which again, with the passage of time, allow the larger to swallow the smaller. Specifically, for one-dimensional surfaces, the size of the parabolic segments increases with the $2 / 3$ power of the time, which is what the numerical simulations suggest.

The noisiness of the problem cannot, in reality, be ignored, but cannot as easily be described in words. But a degree of randomness turns out to be a physical benefit in suggesting analogies with what may happen in other physical circumstances, those of quantum mechanics for example. Indeed, the cleverness of this treatment of the problem of growing surfaces lies in the way it can be transformed from a problem in the solution of differential equations to one much more familiar in quantum electrodynamics, that of describing the timeevolution of a system by the effect of operators on some starting function.

The result, in the surface problem, is a set of simple rules that may describe the simple scaling properties of the problem. That such must emerge follows from the simple observation that, in the case of a one-dimensional surface, a structure which is made up from a sequence of parabolic segments transforms itself in the course of time to another sequence of parabolic segments whose average size has grown by a factor which is some fractional power of the time elapsed. The authors are quick to point out that their model agrees with several of the numerical simulations which have been carried out in circumstances when comparisons are possible. But the importance of this novel treatment is as a physical insight into the aggregation problem when simulations can provide only answers, not understanding. 